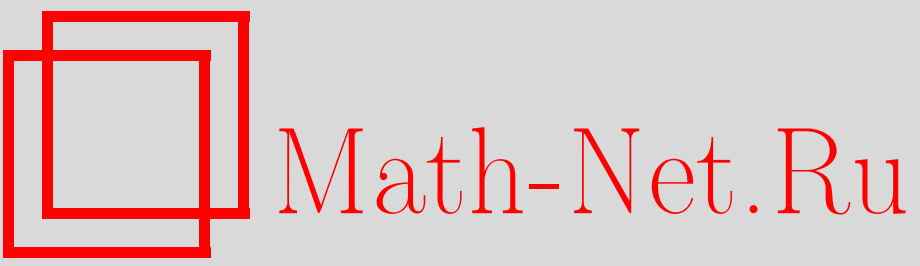

Г. А. Кравцова, В. А. Смирнов, Вычисление трехпетлевых диаграмм Фейнмана с помощью четырехмерного интегрирования по частям и дифференциальной перенормировки, ТМФ, 1997, том 112, номер 1, 115-118

DOI: https://doi.org/10.4213/tmf1031

Использование Общероссийского математического портала Math-Net.Ru подразумевает, что вы прочитали и согласны с пользовательским соглашением

http: //www.mathnet.ru/rus/agreement

Параметры загрузки:

IP : 3.81 .55 .215

26 апреля 2023 г., $17: 11: 46$ 


\section{ВЫЧИСЛЕНИЕ ТРЕХПЕТЛЕВЫХ ДИАГРАММ ФЕЙНМАНА С ПОМОЩЬЮ ЧЕТЫРЕХМЕРНОГО ИНТЕГРИРОВАНИЯ ПО ЧАСТЯМ И ДИФФЕРЕНЦИАЛЬНОЙ ПЕРЕНОРМИРОВКИ}

\footnotetext{
Четырехмерное интегрирование по частям в сочетании с дифференциальной перенормировкой применяется для вычисления трехпетлевых диаграмм Фейнмана.
}

1. В работе [1] было продемонстрировано, как четырехмерное интегрирование по частям в совокупности с техникой дифференциальной перенормировки [2-4] и ее инфракрасного аналога $(\widetilde{R}$-операции) может быть использовано для вычисления двухпетлевых фейнмановских диаграмм. В настоящей заметке мы покажем, что этот метод работает и на трехпетлевом уровне и тем самым оказывается конкурентоспособным по отношению к хорошо известному и успешно применяемому на практике методу интегрирования по частям [5] в рамках размерной регуляризации [6].

В следующих двух пунктах мы в качестве примера проведем вычисление типичной скалярной трехпетлевой диаграммы пропагаторного типа, а в заключении сформулируем выводы.

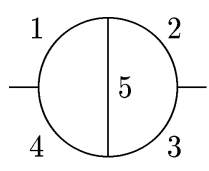

a

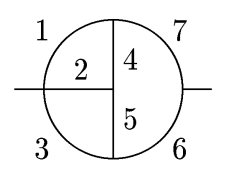

6

Рис. 1. Базисная двухпетлевая диаграмма с двумя произвольными индексами (а) и типичная трехпетлевая диаграмма (б).

2. Рассмотрим сначала базисную двухпетлевую диаграмму с двумя аналитически регуляризованными пропагаторами (рис. 1a):

$$
J(\alpha, 1,1, \beta, 1)=\iint \frac{d^{4} k d^{4} l}{\left(p_{1}^{2}\right)^{\alpha} p_{2}^{2} p_{3}^{2}\left(p_{4}^{2}\right)^{\beta} p_{5}^{2}},
$$

*Московский государственный университет, Москва, Россия. E-mail: galya@class.mi.ras.ru

${ }^{\dagger}$ Московский государственный университет, Москва, Россия. E-mail: smirnov@theory.npi.msu.su 
где $p_{1}=k-q, p_{2}=l-q, p_{3}=l, p_{4}=k, p_{5}=k-l$.

Для случая, когда $\alpha+\beta \neq 2$, используя четырехмерное интегрирование по частям

$$
\iint d^{4} k d^{4} l\left(\frac{\partial}{\partial p_{1}}+\frac{\partial}{\partial p_{4}}+\frac{\partial}{\partial p_{5}}\right) p_{5} I(\alpha, 1,1, \beta, 1)=0,
$$

где $I(\alpha, 1,1, \beta, 1)$ - подынтегральное выражение в (1), получаем

$$
\begin{aligned}
J(\alpha, 1,1, \beta, 1)= & \frac{1}{2-\alpha-\beta} \iint d^{4} k d^{4} l\{\alpha[I(\alpha+1,1,1, \beta, 0)-I(\alpha+1,0,1, \beta, 1)]+ \\
& +\beta[I(\alpha, 1,1, \beta+1,0)-I(\alpha, 1,0, \beta+1,1)]\} .
\end{aligned}
$$

Интегралы от отдельных слагаемых имеют ультрафиолетовые или инфракрасные расходимости. Прежде чем представить интеграл от суммы как сумму интегралов, можно заблаговременно устранить эти расходимости с помощью дифференциальной перенормировки и ее инфракрасного аналога, что было предложено в [1]. Полученные в результате интегралы вычисляются с использованием перенормированного (и $\widetilde{R}$-нормированного) варианта формул однопетлевого интегрирования: например [1]

$$
\widetilde{R} \int d^{4} k \frac{1}{k^{4}(q-k)^{2}}=\pi^{2} \frac{1}{q^{2}}\left(1+\ln q^{2} / \tilde{\mu}^{2}\right) .
$$

В результате получаем известное выражение

$$
\begin{aligned}
& J(\alpha, 1,1, \beta, 1)=\frac{\pi^{4}}{2-\alpha-\beta} \frac{1}{\left(q^{2}\right)^{\alpha+\beta-1}} \frac{\Gamma(1-\alpha) \Gamma(1-\beta) \Gamma(\alpha+\beta-1)}{\Gamma(\alpha) \Gamma(\beta) \Gamma(3-\alpha-\beta)} \times \\
& \quad \times\{(1-\beta)[\psi(\beta)+\psi(2-\beta)-\psi(3-\alpha-\beta)-\psi(\alpha+\beta-1)]+\{\alpha \leftrightarrow \beta\}\} .
\end{aligned}
$$

Соответствующий результат при $\alpha+\beta=2$ можно получить предельным переходом в (5). Однако возможно использование четырехмерного интегрирования по частям и дифференшиальной перенормировки непосредственно при этих значениях параметров исходя из тождества, аналогичного (2), но с дополнительным логарифмом, входящим после производных.

3. Вычислим теперь нашим методом типичную трехпетлевую диаграмму, изображенную на рис. 16 ,

$$
J(q)=\iiint d^{4} k d^{4} l d^{4} r I\left(p_{1}, \ldots, p_{7}\right) .
$$

Как и в двухпетлевом случае [1], начнем с интегрирования по частям со вспомогательным логарифмом:

$$
\iiint d^{4} k d^{4} l d^{4} r\left(\frac{\partial}{\partial p_{1}}-\frac{\partial}{\partial p_{2}}-\frac{\partial}{\partial p_{4}}\right) p_{4} \ln \left(p_{1}^{2} / \mu^{2}\right) I\left(p_{1}, \ldots, p_{7}\right)=0 .
$$

В результате исходная диаграмма оказывается выраженной согласно соотношению, изображенному на рис. 2 , где расходимости диаграмм в правой части устранены с помошью дифференциальной перенормировки. Как и раньше, эти расходимости устраняются, перед тем как полученный интеграл разбивается на сумму от интегралов. 


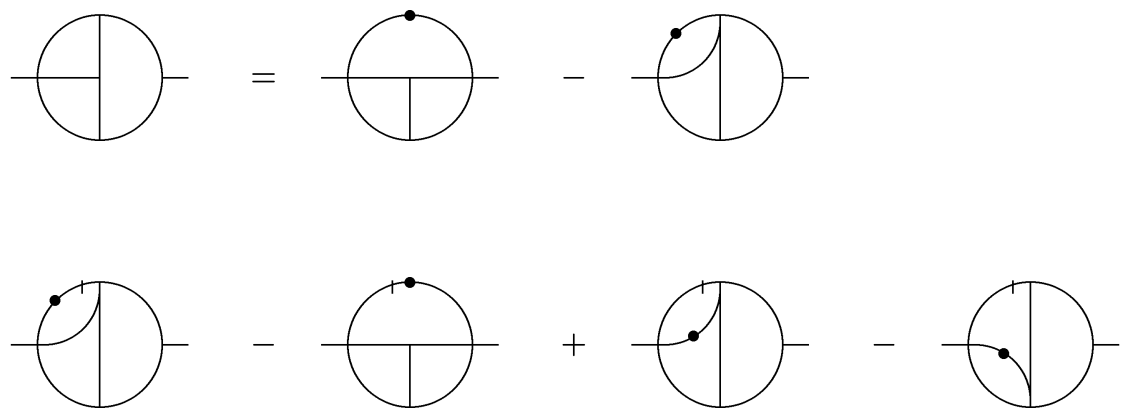

Рис. 2. Вычисление диаграммы, приведенной на рис. $1 б$.

На рис. 2 точка на линии указывает на вторую степень соответствующего пропагатора, а штрих - на наличие логарифма.

Шесть диаграмм в правой части вычисляются с помощью формулы (5), а также формул однопетлевого интегрирования

$$
\begin{gathered}
J_{1}=J_{2}=6 \zeta(3)\left(1+\ln \frac{q^{2}}{\tilde{\mu}^{2}}\right) \frac{\pi^{6}}{q^{2}}, \\
J_{3}=\left\{6 \zeta(3)\left(1+\ln \frac{q^{2}}{\mu^{2}}\right)+3 \zeta(3) \ln \frac{q^{2}}{\tilde{\mu}^{2}}\left(\ln \frac{\tilde{\mu}^{2}}{\mu^{2}}+\ln \frac{q^{2}}{\mu^{2}}\right)+10 \zeta(5)\right\} \frac{\pi^{6}}{q^{2}}, \\
J_{4}=\left\{6 \zeta(3)\left(1+\ln \frac{q^{2}}{\mu^{2}}\right)+3 \zeta(3) \ln \frac{q^{2}}{\tilde{\mu}^{2}}\left(\ln \frac{\tilde{\mu}^{2}}{\mu^{2}}+\ln \frac{q^{2}}{\mu^{2}}\right)\right\} \frac{\pi^{6}}{q^{2}}, \\
J_{5}=\left\{6 \zeta(3) \ln \frac{q^{2}}{\mu^{2}}\left(\ln \frac{q^{2}}{\tilde{\mu}^{2}}+1\right)+20 \zeta(5)\right\} \frac{\pi^{6}}{q^{2}}, \\
J_{6}=\left\{6 \zeta(3) \ln \frac{q^{2}}{\mu^{2}}\left(\ln \frac{q^{2}}{\tilde{\mu}^{2}}+1\right)+10 \zeta(5)\right\} \frac{\pi^{6}}{q^{2}} .
\end{gathered}
$$

Подставляя эти выражения в (6), приходим к хорошо известному результату [7]

$$
J=J_{1}-J_{2}+J_{3}-J_{4}+J_{5}-J_{6}=20 \zeta(5) \frac{\pi^{6}}{q^{2}} .
$$

4. Рассмотренные примеры показывают, что четырехмерное интегрирование по частям в сочетании с дифференциальными $R$ - и $\widetilde{R}$-операциями оказывается вполне удобным методом вычисления фейнмановских диаграмм. Напомним, что любая трехпетлевая безмассовая пропагаторная диаграмма вычисляется в рамках размерной регуляризации с помошью интегрирования по частям [5] за исключением двух диаграмм, для которых применяются дополнительные методы, например техника полиномов Гегенбауэра в координатном пространстве [7]. Ситуация с применением четырехмерного интегрирования по частям выглядит совершенно аналогично, причем соответствуюшие выкладки оказываются того же уровня сложности, что и при использовании интегрирования по частям в размерной регуляризации. Однако для применений в теориях с киральной и суперсимметриями явно четырехмерная техника должна оказаться более предпочтительной. 
Предлагаемая вычислительная техника сочетается с дифференциальной перенормировкой. Хотя общая проблема совместности этой перенормировочной схемы с калибровочной инвариантностью остается открытой, в случае абелевых теорий сушествует вариант дифференциальной перенормировки [4], в рамках которой калибровочная инвариантность обеспечивается автоматически, ${ }^{1)}$ т.е. без "подгонки" конечных контрчленов.

Эта работа поддержана РФФИ, грант 96-01-00654.

Мы благодарны К. Г. Четыркину за ценные обсуждения.

\section{Список литературы}

[1] В. А. Смирнов. ТМФ. 1996. Т. 108. С. 129.

[2] D. Z. Freedman, K. Johnson, J. I. Latorre. Nucl. Phys. B. 1992. V. 371. P. 353; J. I. Latorre, C. Manuel, X. Vilasís Cardona. Ann. Phys. 1994. V. 231. P. 149; V. A. Smirnov. Nucl. Phys. B. 1994. V. 427. P. 325.

[3] О.И. Завьялов, В. А. Смирнов. ТМФ. 1993. Т. 96. С. 288; О. И. Завьялов. ТМФ. 1994. T. 98. C. 536; V. A. Smirnov. Z. Phys. C. 1995. B. 67. S. 531.

[4] V. A. Smirnov. hep-th/9605164; Int. J. Mod. Phys. A. 1997. (in print).

[5] K. G. Chetyrkin, F. V. Tkachov. Nucl. Phys. B. 1981. V. 192. P. 159.

[6] G. 't Hooft, M. Veltman. Nucl. Phys. B. 1972. V. 44. P. 189; C. G. Bollini, J. J. Giambiagi. Nuovo Cim. B. 1972. V. 12. P. 20.

[7] K. G. Chetyrkin, A. L. Kataev, F. V. Tkachov. Nucl. Phys. B. 1980. V. 174. P. 345.

[8] О.И. Завьялов, Г.А. Кравцова, А. М. Малокостов. ТМФ. 1996. Т. 107. С. 64.

Поступила в редакцию 14.II.1997 г.

\section{G. A. Kravtsova, V.A. Smirnov \\ CALCULATION OF THREE LOOP FEYNMAN GRAPHS BY USING FOUR-DIMENSIONAL INTEGRATION BY PARTS AND DIFFERENTIAL RENORMALIZATION}

Four-dimensional integration by parts and differential renormalization technique are applied for computing three loop Feynman graphs.

\footnotetext{
${ }^{1)}$ См. также вариант дифференциальной перенормировки в работе [8], где доказаны соотношения, обеспечивающие выполнимость тождеств Уорда в квантовой электродинамике для некоторых специальных классов диаграмм.
} 